\title{
BMJ Open Reflecting on experiences of care: an exploratory qualitative descriptive study of the perspectives of stroke survivors, families and staff
}

\author{
Caroline Bulsara (D) , ${ }^{1,2}$ Rosemary Saunders (D) , ${ }^{3}$ Laura Emery, ${ }^{3}$ \\ Christopher Etherton-Beer (1) ${ }^{4,5}$
}

To cite: Bulsara C, Saunders R, Emery L, et al. Reflecting on experiences of care: an exploratory qualitative descriptive study of the perspectives of stroke survivors, families and staff. BMJ Open 2021;11:e047559. doi:10.1136/ bmjopen-2020-047559

- Prepublication history and additional supplemental material for this paper are available online. To view these files, please visit the journal online (http://dx.doi.org/10.1136/ bmjopen-2020-047559).

Received 03 December 2020 Accepted 18 November 2021

Check for updates

(c) Author(s) (or their employer(s)) 2021. Re-use permitted under CC BY-NC. No commercial re-use. See rights and permissions. Published by BMJ.

For numbered affiliations see end of article.

Correspondence to Professor Caroline Bulsara; caroline.bulsara@nd.edu.au

\section{ABSTRACT}

Objective The aim of this study was to identify barriers and enablers from the perspectives of stroke survivors, carers and staff to understand the experiences of care. Design The study used a qualitative descriptive methodology and employed semistructured interview technique.

Setting A metropolitan stroke rehabilitation unit in Western Australia providing rehabilitation services for inpatients and outpatients.

Participants Overall, 10 participants (four staff, four stroke survivors and two primary carers) were interviewed. Transcripts were analysed using thematic analysis. Results Experiences of care focused on lack of time, urgency to regain mobility, postshock recovery, uncertainty about the future and the importance of accepting help once home. There was a degree of mismatch between staff experiences of the reality of what can be provided and the experiences and expectations of stroke survivors and families. However, the benefits of a specialised rehabilitation unit were found to contribute to a positive patient experience overall. The specialised unit demonstrated that services must optimise staff time with patients and carers in the poststroke rehabilitation journey to ensure benefits for the long-term well-being for both. Conclusion Seeking patient, family and staff experiences of care can provide valuable insights into facilitating better patient, family and staff engagement for preparation for home-based rehabilitation for stroke survivors and their caregivers. Further research with a larger sample across diverse hospital settings would provide even greater insight into strategies to best address the reality of rehabilitation care and readiness of patients when returning home to the community.

\section{INTRODUCTION}

Stroke is a leading cause of death and disability globally with the burden of stroke the greatest on people under 70 years. ${ }^{1}$ Almost $35 \%$ of Australians who experienced a stroke are left with some resultant disability, 64\% need assistance with healthcare, $58 \%$ with mobility and $47 \%$ with self-care. ${ }^{2}$ Thus, there remains an evident need to provide adequate
Strengths and limitations of this study

- The balance of in-depth opinions between a small sample of hospital staff and stroke survivors and carers provided a more holistic picture of the convergences and contrasts of a patient rehabilitation journey in a single specialised stroke rehabilitation unit.

- This study was a small, exploratory qualitative study but although the sample was small, we did reach saturation.

- The sample represented stroke survivors, family carers and staff from a single specialist rehabilitation hospital. Thus, the findings may not be applicable to those of other general hospital populations providing non-specialised stroke rehabilitation care.

support and services after event to individuals who have had a stroke (stroke survivors) and families impacted by stroke due to the physical, cognitive and psychoemotional consequences that usually require adaption to a more restricted lifestyle with some reduction in activities of daily living. ${ }^{2}$

From hospital data collected between 2015 and 2016, there were 37300 acute care hospitalisations in Australia where stroke was the principal diagnosis, which equates to a rate of 134 patients per 100000 population acute care hospitalisations. ${ }^{3}$ The Acute Stroke Clinical Care Standard devised by the Australian Commission on Safety and Quality in Health Care $^{4}$ identifies the pivotal role carers and family members have in the prevention, early recognition, assessment and recovery for stroke survivors. Furthermore, informal carers such as family members and significant others play a substantial role, providing an estimated 1.9 billion hours of unpaid care in Australia. ${ }^{5}$

The sudden onset of stroke and subsequent early days in an acute hospital setting can be 
traumatic for both the stroke survivor and their families. ${ }^{6}$ The initial admission and early days following a stroke can have a profound effect when the individual and their family begin to realise the impact of the event which can be frightening, confusing and distressing for all. ${ }^{6}$

From a psychological perspective, the shorter term posthospitalisation effects of a stroke have been shown to potentially create a sense of vulnerability for both stroke survivors and families due to impacts on basic activities of daily living. ${ }^{7}$ A number of studies indicate that timely recognition of the physical and the psychological impact of stroke can be beneficial for both stroke survivors and their families. ${ }^{5}$ Vulnerability and uncertainty for the future in turn affect the dignity of the person as they struggle to cope with the discontinuity in body, self and roles both within the family and more widely within the community. ${ }^{9}$ Stroke survivors have reported revaluing their role as an ongoing struggle, and discontinuity and uncertainty were characteristic of the adjustment process after stroke. ${ }^{6} 10$

Nonetheless, adjustment to living with the effects of a stroke could be facilitated by the introduction of relevant and timely strategies such as early coordinated multidisciplinary care in a specialist stroke unit to encourage timely recovery. ${ }^{11}$ The Acute Stroke Clinical Care Standard identifies that a "carer of a patient with stroke is given practical training and support to enable them to provide care, support and assistance to a person with stroke' ( $p$ 2). ${ }^{4}$ Research has identified the important role multidisciplinary healthcare team such as within a specialist stroke rehabilitation setting play in supporting and encouraging the stroke survivor and their family to adjust both physically and emotionally. ${ }^{12}$ Due to the challenges, stroke survivors may feel misunderstood by nurses and other health professionals, and by further understanding their expectations and experiences of care it can contribute to improving their recovery. ${ }^{9}$ This is also critical for improving the safety and effectiveness of healthcare delivery and patient-centred care and aligns with partnering with consumers to improve care and patient outcomes. ${ }^{3} 13$

\section{Early interventions after stroke for patient and family}

Given the suddenness of the onset of stroke, there is a potential for the patient and family to leave the hospital setting before being fully prepared for the continuing rehabilitation process at home. ${ }^{14}$ Home-based community care and rehabilitation may allow earlier patient discharge, yet without access to adequate and ongoing support, families are often ill equipped to deal with the challenges of caring for the individual after their stroke. ${ }^{15}$ Stroke survivors can have a variety of unmet needs at discharge and once the person has returned home, including mobility and communication, awareness of recovery potential, interaction with healthcare professionals, medicines and emotional well-being as well as need for information and aspects of social participation. $^{1617}$
For the stroke survivor, changes in life plans and quality of life can result in longer term psychological impacts including mood swings and mental health issues, ${ }^{81819}$ and found that there was a need for better discharge preparation and a further need for ongoing psychological and social support within the community in understanding readmission after stroke. In addition, a study by Kruithof et $a l^{20}$ demonstrated beneficial outcomes for both family and the stroke survivor relating to good discharge planning practice.

The aim of this study was to add to the body of knowledge regarding the crucial need for early and specialist team-based intervention. Such early intervention would seek to identify barriers and enablers from the perspectives of both stroke survivors and allied health teams. This in turn would assist in better understanding what is required to best support families and stroke survivors during rehabilitation. It was anticipated the synthesis of findings would identify any key areas that could potentially be addressed to ensure better hospital care and support for stroke survivors and their families when transitioning back home. Furthermore, the study lends weight to the contention that a team-based approach provided by the specialist stroke unit both during the stay and while transitioning back to the community enables the strokeaffected individual to better adjust to life after stroke.

\section{METHOD}

\section{Methodology}

This study used a qualitative descriptive (QD) methodology which enables the researcher to stay closer to their data and to the surface of words and events. ${ }^{21}$ Interpretation is much less to the fore ${ }^{22}$ but instead is an extensive summation of events as the participants have described them to the researcher without interpretation by the researcher of those said events. QD is a suitable methodology in healthcare research as it helps to focus research questions directly on the experiences of participants. ${ }^{22}$

\section{Setting and sample}

The study was set in a metropolitan stroke rehabilitation unit in Western Australia providing rehabilitation services for inpatients and outpatients. It is a 10-bed unit within a 199-bed community hospital in Western Australia offering rehabilitation and acute services for inpatients and outpatients. The unit is staffed by a multidisciplinary team of medical, nursing and allied health who provide support both during the hospital stay and prior to and after the patient is discharged back into the community. Interventions are centred on the needs of patients and their families with the average length of stay being 22 days. This unit also provides patients and their families with community rehabilitation services outside of the hospital setting that include occupational therapy, physiotherapy and social work. In this context, clients accessing inpatient rehabilitation tend to have severe impairments 
that preclude early supported discharge to home-based rehabilitation.

Purposive sampling was employed for patient and carer dyads as well as registered nurses and allied health professionals. Purposive sampling is a non-probability sampling technique which selects the sample based on what is known about the target population in concordance with the aim of the research and recruits those who possess the maximum amount of information about the topic. ${ }^{23}$ The first group were patients who had sustained a stroke and were subsequently discharged from the stroke rehabilitation unit within the previous 6-12 months from commencement of the study (following ethical approvals) and met the following criteria:

- Absence of comorbidities such as advanced dementia, cancer or other poor life expectancy conditions that would preclude participation.

- Completion of acute stroke care.

- Over 65 years (or $>45$ years for Aboriginal and Torres Strait Islander people) aligned with the criteria for admission to the rehabilitation unit.

- Be medically stable (ie, not readmitted to acute care or suffering a concurrent illness that would preclude participation in interviews).

In addition, individuals who self-identified as the primary carer (all were spouses) of stroke survivor participants were also invited to participate either in the same interview as the survivor, or in an individual interview. Inclusion criteria were that the person had to be identified as the primary caregiver of the patient and able to communicate in English to a standard that would enable them to fully participate in an in-depth interview with the researcher.

The second group were staff comprising three registered nurses and an occupational therapist who all worked within the specialised community hospital stroke rehabilitation unit and had each been working within the field of specialisation for more than 6 months.

\section{Recruitment procedure}

Stroke survivors and carers

Stroke survivors and carer participants were informed by a research nurse and clinical team member of the study during inpatient care in the rehabilitation unit and provided with a participant information form and consent form. Participants were required to return the consent form by mailing it to the external researcher using the reply-paid envelope and the researcher then followed up with a telephone call to arrange a suitable time and venue for the interview to take place. Of the six participants, five were interviewed in person and the sixth requested to be interviewed by telephone.

\section{Staff}

Staff within the rehabilitation unit were invited to participate in the study by the clinical nurse specialist staff member during a staff meeting and were asked to complete a consent form if willing to be interviewed by the researcher.

\section{Data collection}

Stroke survivor and carer interviews

The QD methodology employed a semistructured interview technique for all participants with an interview guide (online supplemental appendix 1). A researcher who is not employed by the hospital nor the rehabilitation unit completed the interviews. The interview topic areas for survivors and carers focused on a trajectory from recall of stroke event and subsequent hospitalisation, followed by experiences of the stroke rehabilitation unit and ultimately discharge to return home. The interview guides were informed by an extensive literature review and developed in consultation with the individual research team members who are currently working as clinical staff within the rehabilitation hospital including a specialist clinician and a clinical nurse specialist. Interview schedules were piloted with a clinical nursing staff member who understood and was experienced within the rehabilitation field within the hospital. Former inpatient stroke survivors and carers were invited to discuss their individual hospital experiences in terms of their awareness, knowledge and involvement through nursing staff along with understanding the rehabilitation and recovery trajectories after stroke hospitalisation. Stroke survivors and carers were also invited to discuss the future from a personal perspective in terms of how life and roles may have changed and preparedness to remain within their home after rehabilitation unit stay. The interview content also encompassed thoughts around the available support systems from other friends and family members alongside more structured community services. Interviews lasted for approximately 1 hour.

\section{Interviews with nurses and allied health team}

Staff working within the stroke rehabilitation unit were interviewed around perceptions of the stroke survivor and nominated carer during their stay within the rehabilitation unit and their perceptions of preparation for discharge to the community. Staff were also asked to provide any possible examples of where a stroke survivor had transitioned well and conversely any who did not transition successfully including their perceptions around the differences between them and how these related to the role of the participant. All staff interviews were conducted by telephone at suitable times for the participating staff members. Interviews lasted for approximately $30 \mathrm{~min}$.

It was believed that this approach would represent the perspectives of stroke survivors, carers and staff across the rehabilitation trajectory and from multiple viewpoints.

\section{Data analysis}

QD approach requires the researcher to employ either content or thematic analysis to uncover commonalities across a number of participant interviews rather than focusing on the individual experiences of participants. ${ }^{23}$ 
Qualitative data were analysed using template (framework) thematic analysis and common themes extrapolated initially within participant groups and then cross-comparisons between groups were made. All identifying names and characteristics were removed from the groups. Thematic analysis was undertaken using the QSR NVivo V.11.0 data management software. ${ }^{24}$ The thematic template was broadly based on the interview questions and guided by the QD methodological process for data collection and analysis. All themes and subthemes were decided on by two researchers (CB and RS) with consensus agreed on regarding the final coding framework along with the hierarchical structure of themes. It was decided at that point that saturation had been reached with the number of interviews conducted and results addressed the research question.

\section{Patient and public involvement}

Patients and carers were involved in the study during the data collection phase by participating in the in-depth interviews.

\section{Ethical considerations}

The study used a qualitative approach which sought the personal and in-depth opinions and perceptions of patients and wherever possible their carers alongside those of staff providing care within the specialist stroke rehabilitation unit. As such, great care was taken to adhere to the National Health and Medical Research Council (NHMRC) principles of integrity, justice, beneficence and respect for the participants. First, the interviews were conducted by a highly experienced qualitative researcher who was not employed by the hospital and who has expertise in interviewing persons with cognitive impairment and those in vulnerable populations. The team researchers have extensive knowledge of the study population with one of the coauthors being a geriatrician who specialises in stroke outcomes for the older population. The participants were not contacted directly by the researcher interviewer and were invited to make contact with her if they chose to participate. The participants were stroke survivors and carers who had not recently experienced a severe stroke within the last 2 years of the interview period and were community-dwelling individuals. All participants were provided with a participant information sheet which provided details of counselling contact details and an ethics representative number from the university.

\section{RESULTS}

\section{Overarching themes and trajectory of recovery}

Only two carers (one female and one male) of four participating stroke survivors agreed to be interviewed with the survivor to provide supporting detail to the survivor's narrative. Stroke survivor ages ranged from 65 to 84 years. Carer ages were 66 and 86 years.

Overarching themes from the analysis of the interview data from the staff participants centred on staff perceived challenges in rehabilitating stroke survivors to return to the community given the limited available time along with the issues in dealing with families of stroke survivors. Patients and carers highlighted the sudden onset of stroke and the limited adjustment time along with a loss of independence.

Another overarching theme for all three groups was the benefit of providing stroke rehabilitation dedicated facilities along with staff perceptions of the key characteristics of positive and negative readjustments for families of those affected by severe stroke. Figure 1 shows the trajectory of recovery and factors which impact a successful recovery based on this study findings.

\section{From acceptance to adaptability: steps to success}

The themes below highlight the key issues for patients, carers and staff during the rehabilitation phase of the

\section{Trajectory of recovery post stroke}

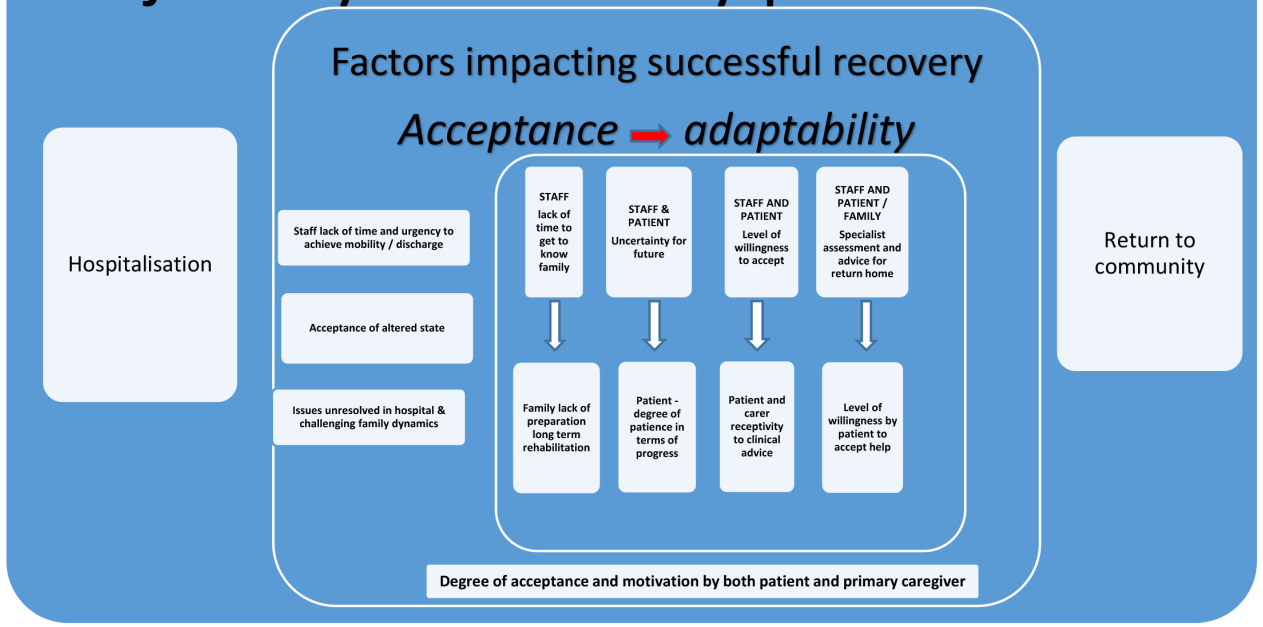

Figure 1 Trajectory of recovery after stroke-staff and patient perspectives. 
recovery journey. Overarchingly, it was evident that there was a mismatch between staff expressing desire to spend more time with the inpatient stroke survivors and families and the actual lack of available time tending to individual patients. Subsequently, this led to unresolved issues for both families and the stroke survivor participant in terms of acceptance and receptivity to clinical advice. The findings below relate to each of the stages of patient rehabilitation and recovery after stroke and show the subthemes of staff perceived lack of time and resultant lack of future preparedness for the stroke survivor and family. Nonetheless, the process for patients and carers in having the ability to adapt was uncovered in the subthemes of willingness to cooperate with staff alongside the level of acceptance for the stroke survivor and family which could potentially be ameliorated by intervention and support of rehabilitation staff.

\section{Lack of time and urgency to regain mobility}

Staff respondents felt that the lack of time to spend with individual patients impacted on the degree of preparation for both the stroke survivor and their family in returning home. This was compounded by an unspoken 'sense of urgency' to 'get the person mobile' again during their hospitalisation in rehabilitation. During the average stay in the rehabilitation unit, staff also encountered patient and family shock and lack of awareness of the level of impact from the stroke event.

It is described as a catastrophic event. So it really is quite life changing-you know-it involves a lot. I think probably the surprise. I mean.... I think the family get a surprise just how much is involved. (Staff participant)

The dynamics of families were also noted as difficult to deal with in the short space of time within the rehabilitation period, with respondents perceiving that there was a mismatch between the hopes and desires of the stroke survivor and the ability of the family to commit to care in a short time frame.

And if you don't have the family support which often they don't because, you know what it's like? You get some families where the children want them to go in a home and they want to go home to their own personal home. So, you've got all those things you have to work through. (Staff participant)

From the stroke survivor and carer perspective, there was some confusion around the initial diagnosis of a severe stroke and a lack of awareness and preparation for potentially what could be a very long-term recovery process.

When we took him to the hospital and they were asking what happened I had already picked it up. I knew....his speech was slurring. But they were still saying that he hadn't had a stroke. But then it might have been just about to happen. (Family participant)
One stroke survivor respondent spoke about the challenge of processing that they had been diagnosed with severe stroke and subsequent loss of mobility (especially loss of independence). This resultant sense of helplessness impacted heavily on the patient's ability to accept and adapt:

I was taking a little water tablet, I also needed to go to the toilet. And that was a nuisance to the nurses. And I got into trouble for that because I got out of bed once. I got out of bed because I was in a room by myself. (Stroke survivor participant)

\section{Postshock recovery and uncertainty about the future}

The findings also highlighted the challenges of the relatively short inpatient rehabilitation time frame given that most staff felt that families were 'in shock'; in the early days and yet having to make major decisions about the stroke survivor's future. The return home also required a period of adjustment both in physical circumstances but also regarding relationships. Staff findings identified an awareness of stroke survivor and family concerns and staff shared that they endeavoured to provide support in whatever ways they could using open communication, reassurance for the stroke survivor and their family as well as providing realism regarding what was possible in terms of a longer term recovery. Nonetheless, the recovery journey was potentially hampered by differences in information and communication expectations between patients, carers and staff and lack of time to process the event.

A very experienced nurse was telling me that we don't allow time for people to grieve ... But also the significant grieving and particularly with some people... I think have worked hard their whole life, and been responsible, and contributing and then for this to happen. (Staff participant)

Staff participants spoke of the characteristics of stroke survivors and carers in terms of adjustment and acceptance. Unwillingness of family members to 'accept reality' was noted as a major issue for staff in trying to emphasise to families what the barriers to providing care within the home are likely to be

Some people are not ready to hear what we are telling them. (Staff participant)

I think sometimes that's where there's-you know-I think sometimes people aren't ready to hear what we are telling them in terms of the extent of the deficits are. (Staff participant)

Despite the fact that staff participants believed that they did their best to reassure inpatient stroke survivors and respond to queries, family support was also clearly important to the acceptance and adaptability process. A participant acknowledged that his wife was well supported through family meetings with the rehabilitation team. 
... if you have any questions then just ask us now. That was good to have the family meeting and then if anybody there had any questions then they could ask and so my daughter, yes she had quite a few concerns and so she was able to ask them. (Family participant)

\section{Returning home and the importance of accepting help}

One of the key advantages of the specialised stroke rehabilitation unit was perceived as the multidisciplinary team who supported the stroke survivor while in hospital as well as assessing the person's living arrangements after returning home. This provided a continuity of care which positively impacted the carer and stroke survivor's ability to return home.

As part of the care in the unit, staff reported that the stroke survivor and the family were prepared for the transition home through inpatient assessment to determine the stroke survivor needs and home assessment to determine if modifications or aids were needed to support the stroke survivor on return to their home. The "wrap around' services before and after discharge supported the continuity of care model offered by the unit.

I guess you kinda hope you covered all those bases before they go home. That you have put enough services and you have put enough support and that they really do know what they are going to deal with. (Staff participant)

Participating staff highlighted that the key characteristics of a positive recovery were observed to be stroke survivor and family confidence in being able to adjust, realistic expectations of what degree of functionality could be achieved in the future and good family support:

But another gentleman that springs to my mind again. He was only able to get home and continue that rehab because everyone had a 'can do' attitude with him. 'Give it a go', 'You're gonna give it a go', 'Let's see what happens' ... do you know what I mean? And that instilled confidence in him as well. (Staff participant)

From a stroke survivor and carer perspective, those interviewed believed that the staff at the specialist rehabilitation unit had facilitated a smooth transition home for the most part. Interestingly, the ability to adjust once home was influenced by the personality and perspective of the patient. The three families who were better equipped emotionally and physically had a primary carer (usually their spouse) to support them. However, sometimes the expectation that participants placed on their children and families was at odds with the reality of the support that significant others were willing and able to provide.

I do get depressed sometimes because I think that I have two sons and daughter here, but our next door neighbours do more for us than them. (Stroke survivor participant)
A participant who had no spouse or partner to provide informal support and care spoke of his frustration with the services and in making himself understood due to speech problems as an outcome of the stroke. Regardless, this participant who lived alone aside from regular visits by his adult son spoke of his resilience and autonomy which he perceived as important in managing his condition. As can be seen in the following quote, the participant who lived alone with visits from his family and neighbours took a sense of pride in his resilience, despite his frustration around speech difficulties.

I am able to look after myself. I've been on my own for 30 years. Important to me. I am strong-I used to work on the oil rigs. (Stroke survivor participant)

In terms of accepting help, participating stroke survivors and carers highlighted the fact that they appreciated help from family and neighbours, but this needed to be balanced to avoid too much intrusion into their personal lives.

He'll [neighbour] pop in every two or threeweeks, [and say] 'everything alright? Is there anything you need?" They're not always in and out of the house. They're not those kinds of neighbours. (Stroke survivor participant)

\section{DISCUSSION}

Summary of main findings and comparison to existing studies The findings from this study highlight the importance of seeking and understanding stroke survivors, family and staff experiences of care to provide quality care for best patient outcomes and prevent readmission. In this study, there were observable differences in experiences in the stroke unit and this identified the mismatch between the reality of what rehabilitation staff can provide and what is expected of them by both stroke survivors and family. In addition to this, there was a limited time frame during hospitalisation for the necessary acceptance of both stroke survivors and family as to the realities of life for the person affected by severe stroke.

These important findings were triggers for nursing and other staff to improve communication with patients and families. Positive experiences of care in other studies were related to reciprocal communication and information sharing with patients and families. ${ }^{13}$

Nurses and health professionals need to understand the complexity in terms of family dynamics and relationships which can be significantly and negatively affected by a sudden illness or traumatic events. ${ }^{2526}$ In this study, it highlighted the issues juxtaposed between staff urgency for the patient to be mobile and ready for discharge, while the stroke survivors and their families were still coming to terms with the shock of the stroke and associated aftereffects thereof. Therefore, good interprofessional skills and providing emotional support is another key part of supporting a positive experience and for nursing staff 
and allied health professionals to better prepare both the stroke survivor and their families in the early rehabilitation days. The early days and the rehabilitation processes were strongly influenced by the stroke survivors and their family members' ability to adjust and maintain focus and positivity. However, for this to be achieved, healthcare team support and communication is essential. ${ }^{27}$

Our findings were in keeping with other studies which indicated that the patient and carers experience two crisis moments following the stroke event. First, the crisis at the time of the stroke and again prior to discharge when the reality of the recovery journey ahead is realised by both the patient and family members. ${ }^{10}$ The second potential crisis point also requires the support of rehabilitation staff members as the reality of discharge 'looms large'. ${ }^{10}$

In terms of early rehabilitation of stroke survivors, the multidisciplinary team are a key part of the provision of care of patients after stroke and supporting their families. The study found that staff identified stroke survivors and family confidence in being able to adjust contributed to a positive transition to home. This reinforces the importance of health professionals conducting a comprehensive assessment of family carer concerns and abilities to support the stroke survivor at home; developing a plan in partnership with stroke survivor and family as part of preparation and support for a caregiving role. ${ }^{28}$

\section{Strengths and limitations}

This study was a small, exploratory qualitative study of the challenges of the recovery during rehabilitation and transition to home from the perspectives of hospital staff, stroke survivors and family carers. Although the sample was small, we did reach saturation for the specific smaller specialised rehabilitation unit. However, the authors recognise that the participant experiences within a small purpose-built stroke rehabilitation unit cannot be generalised to an entire population of survivors of severe stroke. Nonetheless, the balance of opinions between the small specialised rehabilitation team and the patients and carers revealed some core issues around the mismatch of expectations in terms of return to normalcy for patients which were consistent across all interviews. The interviewer is an experienced qualitative researcher and is familiar with interviewing vulnerable populations and those with cognitive impairment. However, the interviewer was not a member of the specialist team nor affiliated with the hospital in any way. This enabled both staff and the patient and carer dyads to be completely free to express forthright opinions confidentially.

Another strength was the inclusion of carers where possible in the study as the carer interviews provided a richer viewpoint regarding the experiences of the patient during the rehabilitation journey. When interviewed together with the stroke survivor, the stories became richer as each participant provided greater detail to the experience of the other.

As the sample represented stroke survivors, family carers and staff from hospital thus may not be applicable to those of other hospital populations such as larger hospital with generalised rehabilitation services. This unit with a coordinated small team of health professionals is specific to providing rehabilitation support for stroke survivors only from inpatient rehabilitation through to return to community and ongoing support once the patient is at home. However, the authors believe that balance of opinions between staff and stroke survivors and carers provided a more holistic picture of their experiences and the rehabilitation journey in a specialised stroke rehabilitation unit.

\section{Implications for clinical practice}

The structure of the rehabilitation multidisciplinary team at the unit demonstrated a willingness to work together to prepare the patient for discharge both within the unit and at the person's home setting. Notwithstanding this ideal, nursing staff participants reported they are under significant pressure to discharge patients once they regain mobility as well as supporting family members and carers, while relying on allied health staff such as occupational therapists and social workers to facilitate their transition home.

\section{CONCLUSION}

As an unforeseen event, stroke can put family members into an unexpected carer role and the findings of this study highlight the importance of staff understanding experiences of care so this can contribute to reflective practice and to 'frank and open' discussion about what is or is not possible and to prepare families and the patient about the pace of rehabilitation. Given the benefits for the long-term well-being of both the stroke survivor and family carer, it is essential that services optimise staff time spent with the individual patient and family in the poststroke rehabilitation journey. This would serve to clarify expectations and reassure stroke survivors and families regarding what to expect in the future once they are discharged from hospital and improve care experiences. Future research exploring how discharge planning and processes for stroke survivor, the education and resources provided to family carers impact on experiences of care and the transition to home and the well-being of the family carer could provide valuable insight into identifying resources to support family carers within limited rehabilitation time frames.

\section{Author affiliations}

${ }^{1}$ School of Nursing and Midwifery, The University of Notre Dame Australia,

Fremantle, Western Australia, Australia

${ }^{2}$ Insitute for Health Research, The University of Notre Dame Australia, Fremantle, Western Australia, Australia

${ }^{3}$ School of Nursing and Midwifery, Edith Cowan University, Joondalup, Western Australia, Australia

${ }^{4}$ School of Medicine and Pharmacology, The University of Western Australia, Perth, Western Australia, Australia

${ }^{5}$ WA Centre for Health and Ageing, The University of Western Australia, Perth, Western Australia, Australia 
Acknowledgements The authors would like to acknowledge the willingness of patients, carers and staff of the specialised stroke rehabilitation unit who gave generously their time to share their stories and rehabilitation journeys.

Contributors CB developed the methodology for the study, conducted the data collection, analysed the data and drafted the manuscript. RS analysed the data and contributed significantly to drafting of the manuscript. LE drafted the manuscript and prepared the manuscript for publication. CE-B developed the study concept and contributed to the manuscript. CB is the author responsbile for overall content and accepts full responsibility for the work, had full access to data and controlled decision to publish.

Funding This work was supported by an internal research grant scheme of the University of Notre Dame Australia (grant number 2016R11116).

Competing interests None declared.

Patient consent for publication Not required.

Ethics approval This study involves human participants and was approved by The University of Notre Dame Research Ethics Committee (reference number: 016175F). Participants gave informed consent to participate in the study before taking part. This study received ethical approval (2016-36; 14 December 2016) from both The University of Notre Dame Australia Human Research Ethics Committee (HREC) and the South Metropolitan Health Service HREC in Perth, Western Australia.

Provenance and peer review Not commissioned; externally peer reviewed. Data availability statement № data are available.

Supplemental material This content has been supplied by the author(s). It has not been vetted by BMJ Publishing Group Limited (BMJ) and may not have been peer-reviewed. Any opinions or recommendations discussed are solely those of the author(s) and are not endorsed by BMJ. BMJ disclaims all liability and responsibility arising from any reliance placed on the content. Where the content includes any translated material, BMJ does not warrant the accuracy and reliability of the translations (including but not limited to local regulations, clinical guidelines, terminology, drug names and drug dosages), and is not responsible for any error and/or omissions arising from translation and adaptation or otherwise.

Open access This is an open access article distributed in accordance with the Creative Commons Attribution Non Commercial (CC BY-NC 4.0) license, which permits others to distribute, remix, adapt, build upon this work non-commercially, and license their derivative works on different terms, provided the original work is properly cited, appropriate credit is given, any changes made indicated, and the use is non-commercial. See: http://creativecommons.org/licenses/by-nc/4.0/.

\section{ORCID iDs}

Caroline Bulsara http://orcid.org/0000-0003-4482-563X

Rosemary Saunders http://orcid.org/0000-0001-6213-4694

Christopher Etherton-Beer http://orcid.org/0000-0001-5148-0188

\section{REFERENCES}

1 GBD 2016 Stroke Collaborators. Global, regional, and national burden of stroke, 1990-2016: a systematic analysis for the global burden of disease study 2016. Lancet Neurol 2019;18:439-58.

2 Gabel CP, Rando N, Melloh M. Slacklining and stroke: a rehabilitation case study considering balance and lower limb weakness. World $J$ Orthop 2016;7:513-8.

3 Australian Institute of Health and Welfare. Australia's health 2018. Australia's health series no. 16. AUS 221. Canberra: AlHW, 2018.

4 Australian Commission on Safety and Quality in Health Care. 2015). acute stroke clinical care standard. Sydney: ACSQHC, 2015.

5 Australian Institute of Health and Welfare. Australia's welfare 2017. Australia's welfare series no. 13. AUS 214. 2017. Canberra: AlHW, 2017.
6 Cecil R, Thompson K, Parahoo K, et al. Towards an understanding of the lives of families affected by stroke: a qualitative study of home carers. J Adv Nurs 2013;69:1761-70.

7 Lutz BJ, Young ME, Cox KJ, et al. The crisis of stroke: experiences of patients and their family caregivers. Top Stroke Rehabil 2011;18:786-97.

8 Sagen U, Finset A, Moum T, et al. Early detection of patients at risk for anxiety, depression and apathy after stroke. Gen Hosp Psychiatry 2010;32:80-5.

9 Satink T, Cup EH, llott I, et al. Patients' views on the impact of stroke on their roles and self: a thematic synthesis of qualitative studies. Arch Phys Med Rehabil 2013;94:1171-83.

10 Gillespie D, Campbell F. Effect of stroke on family carers and family relationships. Nurs Stand 2011;26:39-46.

11 Lynch EA, Mackintosh S, Luker JA, et al. Access to rehabilitation for patients with stroke in Australia. Med J Aust 2019;210:21-6.

12 Clarke DJ, Forster A. Improving post-stroke recovery: the role of the multidisciplinary health care team. J Multidiscip Healthc 2015;8:433-42.

13 Harrison R, Walton M, Manias E. Patients' experiences in Australian hospitals: an evidence check rapid review brokered by the Sax Institute for the Australian Commission on safety and quality in health care, 2015. Available: www.saxinstitute.org.au

14 Luker J, Murray C, Lynch E, et al. Carers' experiences, needs, and preferences during inpatient stroke rehabilitation: a systematic review of qualitative studies. Arch Phys Med Rehabil 2017;98:1852-62.

15 Lutz BJ, Young ME, Creasy KR, et al. Improving stroke caregiver readiness for transition from inpatient rehabilitation to home. Gerontologist 2017;57:880-9.

16 De Simoni A, Shanks A, Balasooriya-Smeekens C, et al. Stroke survivors and their families receive information and support on an individual basis from an online Forum: descriptive analysis of a population of 2348 patients and qualitative study of a sample of participants. BMJ Open 2016;6:e010501

17 Maclean N, Pound P, Wolfe C, et al. Qualitative analysis of stroke patients' motivation for rehabilitation. BMJ 2000;321:1051-4.

18 Australian Institute of Health and Welfare. Functional Independence Measure. Health, Standard 11/04/2014 - description of FIM, 2019. Available: https://meteor.aihw.gov.au/content/index.phtml/itemld/ 495857

19 White CL, Brady TL, Saucedo LL, et al. Towards a better understanding of readmissions after stroke: partnering with stroke survivors and caregivers. J Clin Nurs 2015;24:1091-100.

20 Kruithof WJ, Visser-Meily JMA, Post MWM. Positive caregiving experiences are associated with life satisfaction in spouses of stroke survivors. J Stroke Cerebrovasc Dis 2012;21:801-7.

21 Sandelowski M. Whatever happened to qualitative description? Res Nurs Health 2000;23:334-40.

22 Neergaard MA, Olesen F, Andersen RS, et al. Qualitative description - the poor cousin of health research? BMC Med Res Methodol 2009;9:52.

23 Polit DFB, Beck CT. Essentials of nursing research: appraising evidence for nursing practice. 7th edn. Philadelphia, 2010.

24 QSR International Pty Ltd. NVivo (version 11.0), 2015. Available: https://www.qsrinternational.com/nvivo-qualitative-data-analysissoftware/home

25 Degeneffe CE, Gagne LM, Tucker M. Family systems changes following traumatic brain injury: adult sibling perspectives. J Appl Rehabil Couns 2013;44:32-41.

26 Keeley AC, Chase L. Effects of mental illness on the family: experiential family assessment to promote students' affective learning. J Nurs Educ 2012;51:111-4.

27 Ellis-Hill C, Robison J, Wiles R, et al. Going home to get on with life: patients and carers experiences of being discharged from hospital following a stroke. Disabil Rehabil 2009;31:61-72.

28 Young ME, Lutz BJ, Creasy KR, et al. A comprehensive assessment of family caregivers of stroke survivors during inpatient rehabilitation. Disabil Rehabil 2014;36:1892-902. 